\author{
К.С. Рогаев, А.Н. Ищенко, В.В. Буркин, А.С. Дьячковский, \\ А.Д. Сидоров, Е.Ю. Степанов \\ ИССЛЕДОВАНИЕ ГОРЕНИЯ ВЫСОКОПЛОТНЫХ ТОПЛИВ
В УСЛОВИЯХ МОДЕЛЬНОЙ БАЛЛИСТИЧЕСКОЙ УСТАНОВКИ
}

Рассмотрено горение высокоплотных топлив в условиях динамических давлений, создаваемых во время выстрела в модельной баллистической установке калибром 30 мм. В рамках проведенного расчетно-экспериментального исследования получен закон диспергирования и горения высокоплотного топлива, используемого в качестве присоединенного заряда. Включение высокоплотных топлив в метательный заряд позволило увеличить дульную скорость метаемого поршня-снаряда в условиях модельной баллистической установки.

Ключевые слова: внутренняя баллистика, ствольные системы, газодинамика, присоединенный заряд, горение топлив, высокоплотные топлива.

Использование классических подходов для повышения дульной скорости метаемого элемента в области ствольной баллистики не позволяет получить существенный прирост. Наличие «эффекта насыщения» ограничивает скорость снаряда в классической схеме выстрела при использовании порохового заряда, поскольку максимальная скорость метания при сгорании заряда определяется предельной скоростью разлета пороховых газов в вакууме. Перспективным является применение нетрадиционных схем заряжания [1-3], в частности схемы с присоединенным зарядом (П3) и комбинированной схемы заряжания [4-8]. Использования данных нетрадиционных схем заряжания позволяет за счет включения в метательный заряд топлив увеличить суммарную массу метательных веществ и как следствие повысить среднюю плотность заряжания. Применение данных схем приводит к перераспределению энергии продуктов горения в заснарядном пространстве, что позволяет снизить максимальное давление в камере заряжания. При использовании схемы с ПЗ появляется возможность реактивного подгона снаряда в стволе. Перечисленные преимущества позволяют повысить дульную скорость снаряда без повышения максимального давления на дно канала ствола.

Для достижения описанных положительных эффектов в качестве ПЗ используют различные варианты топлив, разработанных для каждого конкретного случая, одним из перспективных вариантов является использование высокоплотных топлив. Преимуществом данных топлив является плотность укладки или насыпная плотность (по аналогии с пороховым зарядом) $\Delta \geq 1.5 \Gamma / \mathrm{cm}^{3}$, превышающая насыпную плотность традиционных зерненых пироксилиновых порохов $\left(\Delta \leq 1.0\right.$ г $\left./ \mathrm{cm}^{3}\right)$, что позволяет включить в метательный заряд большее количество метательных веществ или же провести частичную замену традиционного метательного заряда, тем самым увеличить суммарную массу заряда.

\footnotetext{
${ }^{1}$ Исследование выполнено за счет гранта Российского научного фонда (проект № 19-79-00028).
} 
При проектировании новых ствольных баллистических систем, использующих в составе метательного заряда высокоплотные топлива функционирующих в схеме заряжания с присоединенным зарядом необходимо знать механизмы разрушение моноблока из данных топлив на частицы (диспергирование) и горение образовавшихся частиц в послойном режиме в условиях динамических давлений, реализуемых в выстреле.

Проведено комплексное экспериментально-теоретическое исследование законов диспергирования и горения высокоплотных топлив, включенных в метательный заряд, в условиях модельной баллистической установки.

\section{Экспериментальная методика}

Экспериментальные внутрибаллистические исследования проводились на баллистическом стенде [9] НИИ ПММ ТГУ в состав которого входит модульная модельная баллистическая установка. Данная баллистическая установка позволяет изменять конфигурацию ствол-камеры под требуемые параметры исследования. В состав баллистической установки входит вакуумируемая баллистическая трасса с пулеприемником, в котором располагается требуемый набор преград. Внешний вид баллистической установки представлен на рис. 1.

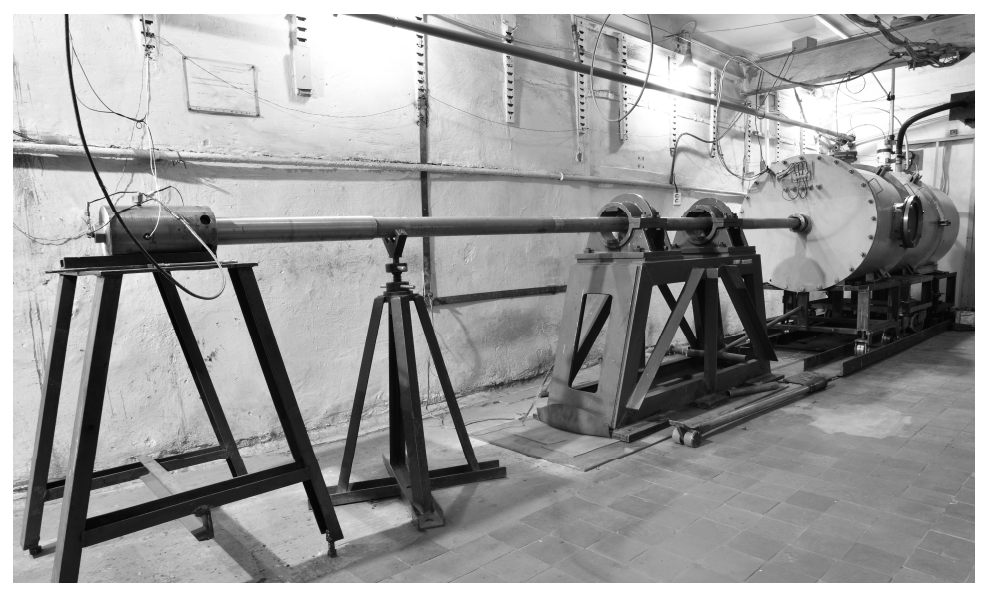

Рис. 1. Внешний вид модельной баллистической установки и вакуумируемой баллистической трассы

Fig. 1. Design of the model ballistic installation and evacuated ballistic track

В состав баллистического стенда входит измерительно-регистрирующий комплекс [10], состоящий из следующего парка оборудования:

- пьезокварцевые датчики 2Т6000 и комплект аппаратуры «Нейва-10000» со специальным программным обеспечением для измерения давления в баллистических установках в диапазоне от 0 до $600 \mathrm{MПа;}$

- индукционные датчики дульной скорости метаемых тел в диапазоне от 100 до $3000 \mathrm{~m} / \mathrm{c}$ [11];

- измерители скорости снаряда в стволе во время выстрела «ДДС-6000» на основе СВЧ-радаров [12];

- осциллографы цифровые запоминающие «Tektronix» DPO2014, «Instek» GDS-810С и «RIGOL» DS7024. 
Во время проведения баллистических испытаний регистрируется: изменение давления в камере заряжания $P(t)$, фиксируется максимальное давление на дно канала ствола $P_{\max }$, временная зависимость скорости снаряда в стволе $V_{\text {сн }}(t)$ и дульная скорость снаряда $V$. Для обеспечения отказоустойчивости лабораторного баллистического стенда предусмотрена дублирующая ветка регистрации основных баллистических характеристик, состоящая из представленных осциллографов.

В качестве снаряда использовался поршень массой 50 г, изготовленный из текстолита и запрессованной в него стальной метки. В качестве метательного заряда использовался одноканальный пироксилиновый порох марки «Сунар 30-06».

Для определения базовых значений дульной скорости проведены эксперименты при использовании классической схемы заряжания, где в качестве метательного заряда применялся только порох. Полученные экспериментальные данные использовались как эталонные для сравнения классической схемы заряжания и схемы с высокоплотными топливами в качестве ПЗ. Внешний вид компоновки выстрела при использовании классической схемы заряжания представлен на рис. $2, a$.
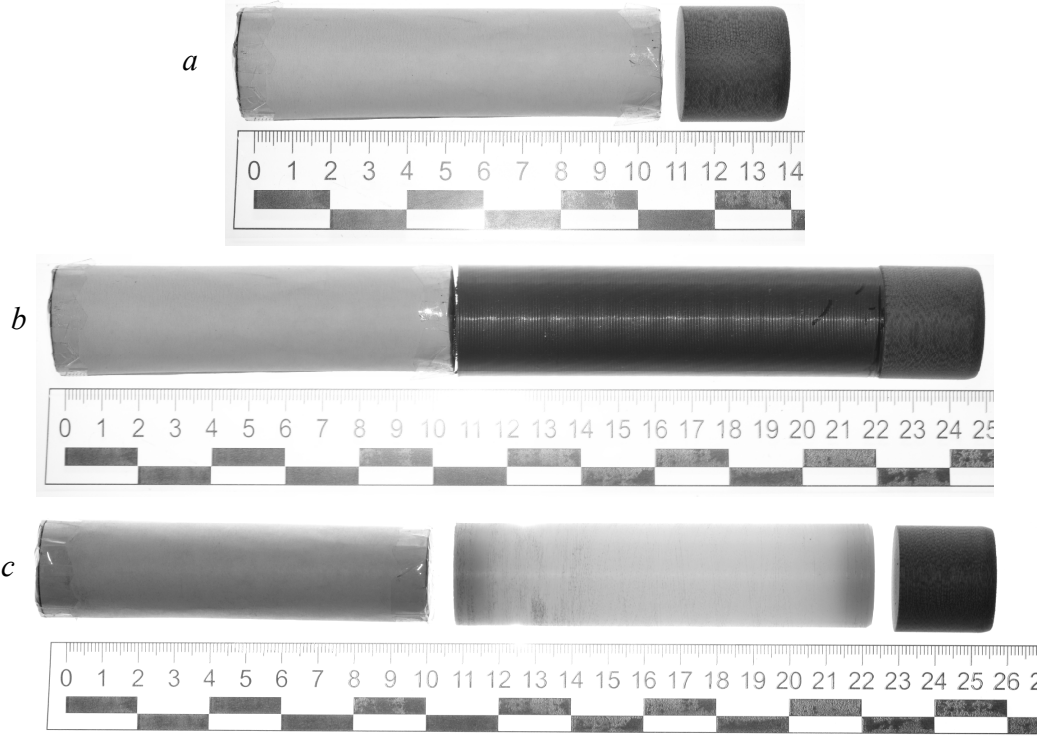

Рис. 2. Внешний вид выстрела: $a$ - классическая схема; $b$ - схема с присоединенным зарядом; $c$ - схема с имитатором ПЗ

Fig. 2. Schematic representation of a shot: $(a)$ a classic scheme, $(b)$ a scheme with a traveling charge (TC), and (c) a scheme with a TC-simulator (TCS)

В качестве ПЗ использовалось высокоплотное топливо массой $m$ и $2 m$ г и размещенное в пластиковом контейнере, изготовленном методом послойного наплавления SBS-пластика. Данный контейнер необходим для предотвращения воспламенения ПЗ по боковой поверхности при движении по стволу. Внешний вид выстрела с ПЗ представлен на рис. 2, c. Контейнеры при использовании $m$ и $2 m$ высокоплотного топлива имели массу 5 и 9 г соответственно.

Для определения начала горения присоединенного заряда, состоящего из высокоплотного топлива, были проведены эксперименты, где в качестве ПЗ использовался имитатор (ИПЗ). ИПЗ изготавливался из полиэтилена, в котором с каждо- 
го из торцов запрессовывалась металлическая шайба, при этом масса и длина были равны ПЗ из высокоплотного топлива (рис. 2, c). Выполнение предварительного эксперимента с ИПЗ соответствующей массы было необходимо для корректного определения навески порохового заряда, обеспечивающего достижение требуемого максимального давления на дно канала ствола при включении высокоплотных топлив в метательный заряд. Результаты проведенных экспериментальных внутрибаллистических исследований, направленных на получение законов горения высокоплотных топлив в условиях динамических давлений представлены на рис. 3.

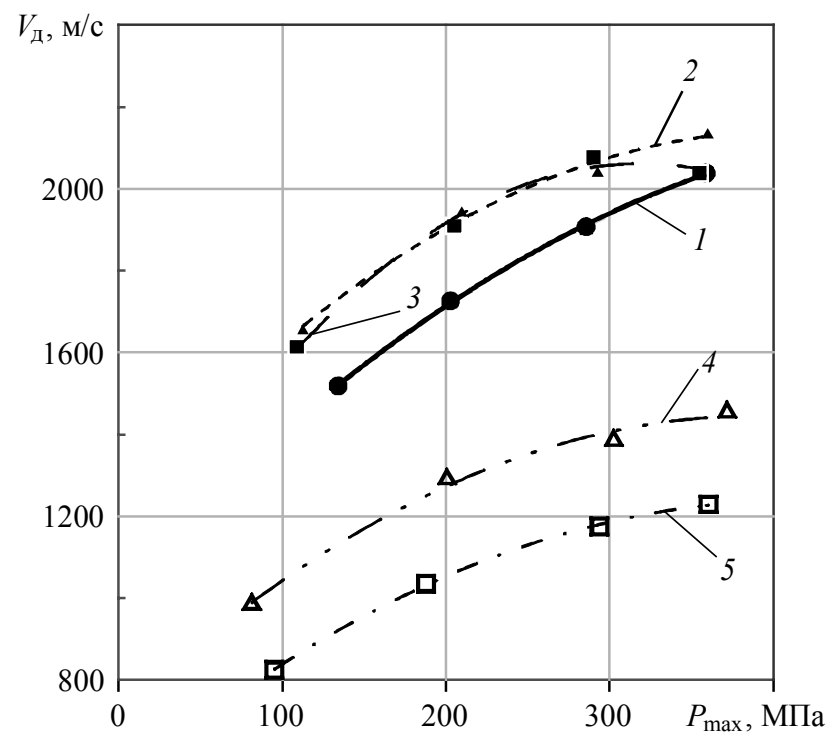

Рис. 3. Зависимость дульной скорости снаряда от максимального давления на дно канала ствола: $1, \bullet-$ классическая схема заряжания; 2, $\boldsymbol{\Delta}$ - схема заряжания с ПЗ массой $m ; 3$, - схема заряжания с ПЗ массой $2 m$; $4, \Delta$ - схема заряжания с ИПЗ массой $m ; 5, \square-$ схема заряжания с ИПЗ массой $2 m$

Fig. 3. Dependence of the projectile muzzle velocity on the maximum of the bore bottom pressure: $1, \bullet-$ a classic loading scheme; 2, $\mathbf{\Delta}$ - a loading scheme with a TC with a mass of $m ; 3,-$ a loading scheme with a TC with a mass of $2 m$; $4, \Delta-$ a loading scheme with a TCS with a mass of $m$; and 5 , $\square-$ a loading scheme with a TCS with a mass of $2 m$

Как показал анализ результатов проведенных экспериментальных исследований, представленных на рис. 3, включение в состав метательного заряда высокоплотных топлив в виде ПЗ позволяет увеличить скорость снаряда на дульном срезе на $(4.9 \div 12.7)$ \% по сравнению с классической схемой заряжания, при сохранении максимального давления на дно канала ствола. Однако при использовании высокоплотного топлива массой $2 m$ и максимальном давлении $P_{\max }=360 \mathrm{MПа} \mathrm{не}$ получаем выигрыша в дульной скорости, это объяснятся тем, что большая часть ПЗ не успела сгореть за время выстрела. 


\section{Расчетная методика}

Все эксперименты были обработаны с применением программного комплекса, разработанного сотрудниками НИИ ПММ ТГУ [13]. В программном комплексе используется математическая модель, которая базируется на основных допущениях механики многофазных сред. В данной модели предусмотрено наличие в метательном заряде произвольного количества фракций частиц и возможность добавления моноблочных элементов (в данном случае высокоплотных топлив), которые диспергируют на отдельные частицы дополнительной фракции и горят по своим законам.

На рис. 4 представлено сравнение расчетно-экспериментальных данных для классической схемы заряжания: для классической схемы заряжания (a) и схемы заряжания с ИПЗ массой $m(b)$ и $2 m(c)$. Как видно из сравнения экспериментальных и расчетных данных, представленных на рис. 4, используемая модель позволяет хорошо описывать процессы в заснарядном пространстве. Расхождение расчетных и экспериментальных данных по максимальному давлению не превышает $3 \%$, по дульной скорости снаряда $-1 \%$. Следовательно, данная математическая модель может быть использована для расчета газодинамической картины при включении в метательный заряд высокоплотных топлив и получения законов его диспергирования и горения.

В рамках использованной математической модели выстрела с ПЗ предполагалось, что зона или фронт горения высокоплотного топлива заменяется поверхностью сильного газодинамического разрыва. Параметры справа и слева от разрыва связывались соответствующими условиями динамической совместности. Предполагалось, что топливо во фронте горения частично сгорает, а частично диспергирует на совокупность одинаковых частиц, догорающих в заснарядном объеме в послойном режиме.

Закон диспергирования присоединенного заряда записывался как степенная зависимость от давления и выглядел следующим образом:

$$
U_{s}=B_{s}\left(\frac{P}{P_{\text {атм }}}\right)^{v},
$$

где $B_{\mathrm{s}}$ - скорость диспергирования при $P=P_{\text {атм }}=0.1$ МПа; $P$-давление; $P_{\text {атм }}-$ атмосферное давление; $v$ - показатель степени (для исследуемого топлива $v=0.8)$.

Наибольший прирост скорости снаряда при использовании ПЗ получается тогда, когда ПЗ загорается позже порохового заряда. В качестве характеристики задержки начала горения моноблока используется импульс давления на фронте го-

рения $I_{1}=\int_{t_{0}}^{t_{1}} P(t) d t$ (импульс начала горения присоединенного заряда). Закон горения моноблочного заряда выглядит следующим образом:

$$
\begin{gathered}
U_{\mathrm{s}}=0, \text { при } I<I_{1}, \\
U_{s}=B_{s 1}\left(\frac{P}{P_{\text {атм }}}\right)^{v} \text {, при } I \geq I_{1} \text { где } I_{1}=\int_{t_{0}}^{t_{1}} P(t) d t .
\end{gathered}
$$

Степень выгорания топлива во фронте горения характеризовалась параметром $\psi_{0}[14]$, который при проведении расчетов принимался равным $\psi_{0}=0.1$. 

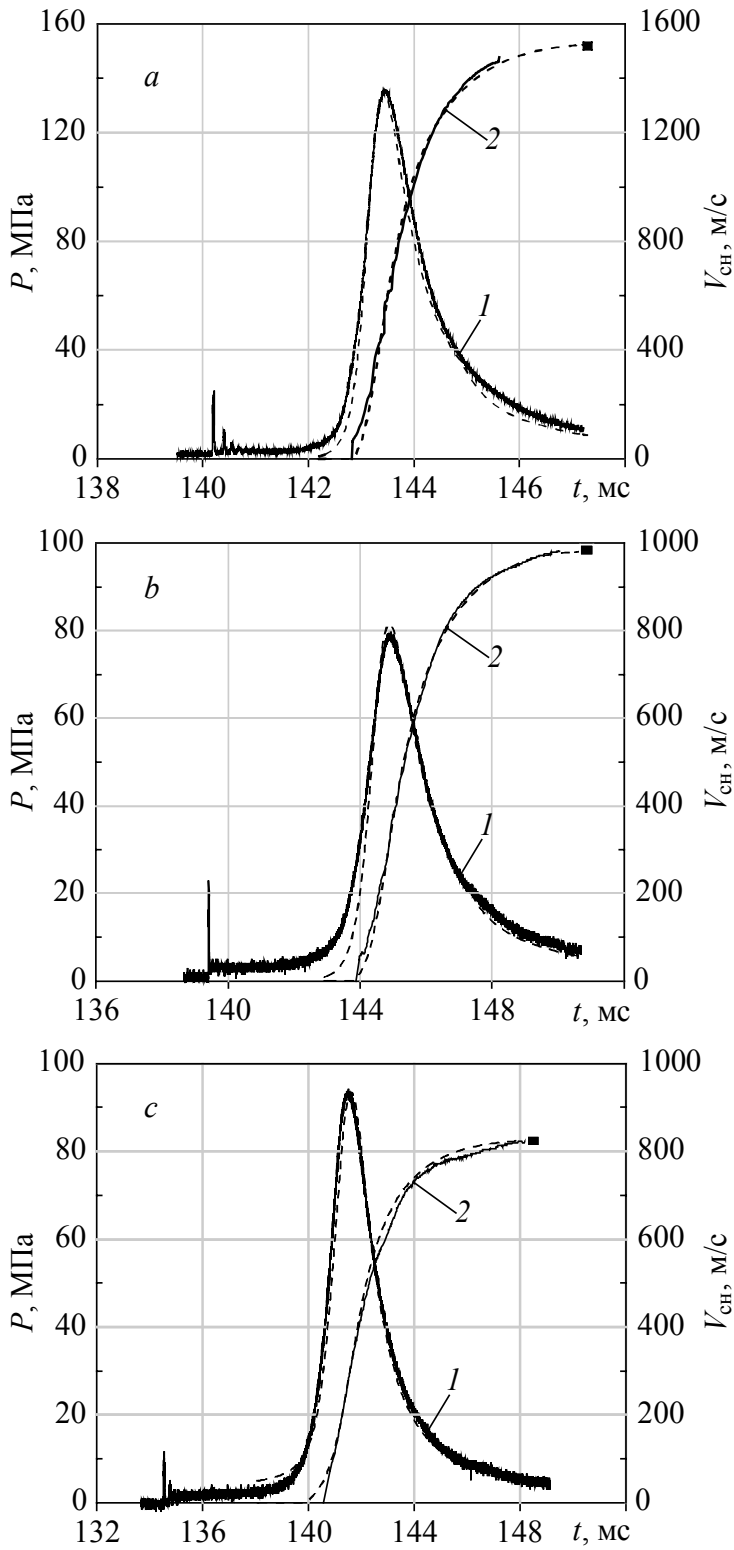

Рис. 4. Временные зависимости давления на дно канала ствола и скорости снаряда в стволе при использовании классической схемы заряжания: $a$ - снаряд 50 г; $b$ - снаряд 50 г + ИПЗ $m ; c$ - снаряд 50 г + ИПЗ $2 m$; 1 - давление на дно канала ствола; 2 - скорость снаряда в стволе; - - дульная скорость снаряда; — - - эксперимент; - - - - расчет

Fig. 4. The pressure on the bore bottom and the projectile velocity in the barrel as the functions of time when using the classic loading scheme: (a) a $50 \mathrm{~g}$ projectile; (b) a $50 \mathrm{~g}$ projectile + TCS with a mass of $m$; and (c) a $50 \mathrm{~g}$ projectile + TCS with a mass of $2 \mathrm{~m}$; 1 , the pressure on the bore bottom; 2 , the projectile velocity in the barrel; - - the projectile muzzle velocity; - - experiment; - - - - calculation 
Послойное горение диспергированных частиц высокоплотного топлива в математической модели при движении по стволу также определялось общепринятой зависимостью от давления [15]:

$$
U_{C}=U_{1}\left(\frac{P}{P_{\text {атм }}}\right)^{v},
$$

где $U_{1}-$ скорость горения при $P=P_{\text {атм }}=0.1$ МПа (зависит от химической природы топлива); $v$ - показатель степени (в расчете $v=0.8)$.

На рис. 5 показан типичный результат сравнения расчетных и экспериментальных данных с использованием $m(a)$ и $2 m(b)$ высокоплотных топлив, в качестве ПЗ.
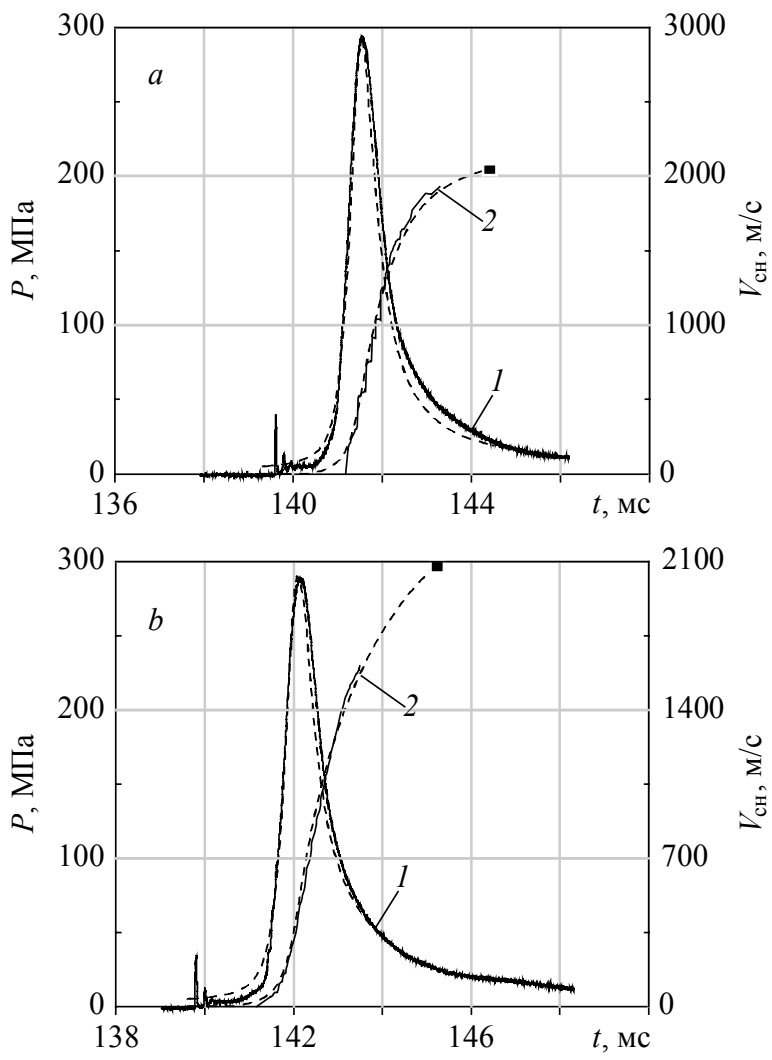

Рис. 5. Временные зависимость давления на дно канала ствола и скорости снаряда в стволе при использовании ПЗ массой $m(a)$ и $2 m(b)$ г с учетом контейнера: 1 - давление на дно канала ствола; 2 - скорость снаряда в стволе; - - дульная скорость снаряда; — - эксперимент; - - - расчет

Fig. 5. The pressure on the bore bottom and the projectile velocity in the barrel as the functions of time when using a TC with a mass of (a) $m$ and (b) $2 \mathrm{~m}$ g taking into account the container: 1 , the pressure on the bore bottom; 2 , the projectile velocity in the barrel; - - the projectile muzzle velocity; —- experiment; - - - - calculation 
Как и в случае с классической схемой заряжания расхождение расчетноэкспериментальных данных по максимальному давлению на дно канала ствола и дульной скорости снаряда составляет не более 3 и 1 \% соответственно. Представленные результаты сравнения расчетно-экспериментальных данных свидетельствуют о получении правдоподобной картины распределения газодинамических параметров в выстреле при использовании высокоплотных топлив.

\section{Результаты параметрических исследований}

Анализ полученных расчетно-экспериментальных данных позволил получить параметры закона диспергирования и послойного горения высокоплотного топлива при использовании перспективной схемы заряжания в условиях динамических давлений, реализуемых в выстреле из модельной баллистической установки. Теоретические результаты по определению законов диспергирования и горения высокоплотных топлив, основанные на экспериментальных данных, представлены в таблице.

\section{Законы горения высокоплотного топлива, полученные теоретическим образом} из обработки экспериментальных данных

\begin{tabular}{|c|c|c|c|c|c|c|c|c|c|}
\hline № & $\omega_{\Pi 13}, \Gamma$ & $\begin{array}{l}P_{\max }, \\
\text { МПа }\end{array}$ & $V, \mathrm{M} / \mathrm{c}$ & $\begin{array}{c}I_{\mathrm{\Pi}}, \\
\mathrm{M \Pi а} \cdot \mathrm{c}\end{array}$ & $\begin{array}{l}B_{1 \mathrm{~s}}, \\
\mathrm{M} / \mathrm{c}\end{array}$ & $\begin{array}{l}x_{\text {кд }}, \\
\mathrm{cM} \\
\end{array}$ & $\begin{array}{c}m_{\text {опз }}, \\
\Gamma\end{array}$ & $\begin{array}{c}U_{1}, \\
\mathrm{MM} / \mathrm{c}\end{array}$ & $m_{\text {опч }}, \Gamma$ \\
\hline 1 & 2 & 3 & 4 & 5 & 6 & 7 & 8 & 9 & 10 \\
\hline 1 & $m$ & 112.7 & 1659 & 80 & 0.19 & 249.9 & - & 0.30 & 12.8 \\
\hline 2 & $2 m$ & 109.1 & 1614 & 85 & 0.19 & - & 8.4 & 0.29 & 25.3 \\
\hline 3 & $m$ & 209.9 & 1945 & 80 & 0.20 & 160.2 & - & 0.30 & 8.6 \\
\hline 4 & $2 m$ & 205.3 & 1909 & 85 & 0.19 & - & 2.5 & 0.29 & 23.8 \\
\hline 5 & $m$ & 292.9 & 2044 & 85 & 0.19 & 181.0 & - & 0.29 & 10.1 \\
\hline 6 & $2 m$ & 290.3 & 2077 & 85 & 0.19 & - & 0.2 & 0.30 & 23.5 \\
\hline 7 & $m$ & 360.0 & 2138 & 85 & 0.19 & 162.6 & - & 0.29 & 9.3 \\
\hline 8 & $2 m$ & 354.9 & 2038 & 85 & 0.17 & - & 5.7 & 0.26 & 24.7 \\
\hline
\end{tabular}

В таблице по столбцам приняты следующие обозначения: 1 - номер эксперимента; 2 - масса присоединенного заряда из высокоплотного топлива без контейнера; 3 - максимальное давление на дно канала ствола; 4 - дульная скорость снаряда; 5 - импульс начала горения присоединенного заряда; 6 - коэффициент в законе скорости диспергирования; 7 - координата снаряда при окончании диспергирования ПЗ; 8 - масса остатка ПЗ, вылетевшего из ствола вслед за снарядом; 9 коэффициент, зависящий от химической природы топлива; 10 - суммарная масса частиц высокоплотного топлива, не сгоревших за время выстрела.

Как показал газодинамический анализ расчетно-экспериментальных данных представленных в таблице, диспергирование присоединенного заряда, состоящего из высокоплотного топлива, начинается с момента $I_{\text {п }} \geq 85 \mathrm{MПа \cdot с,} \mathrm{и} \mathrm{продолжается}$ в течение всего процесса выстрела либо до полного превращения в частицы и газ, либо до вылета из ствола вслед за снарядом. Диспергированное топливо на частицы продолжает гореть при движении по каналу ствола модельной баллистической установки. Однако сильная зависимость послойной скорости горения $(v=0.8)$ не позволяют высокоплотному топливу сгореть полностью за время выстрела.

В результате получено, что законы диспергирования и горения высокоплотного топлива в условиях динамических давлений, реализуемых в модельной баллистической установке во время выстрела, имеют следующий вид: 
диспергирование -

$$
\begin{gathered}
U_{\mathrm{s}}=0, \text { при } I_{\Pi}<85 \mathrm{MПа} \cdot \mathrm{c}, \\
U_{s}=0.019\left(\frac{P}{P_{\text {атм }}}\right)^{0.8} \mathrm{M} / \mathrm{c}, \text { при } I_{\text {п }} \geq 85 \mathrm{MПа} \cdot \mathrm{c} ;
\end{gathered}
$$

послойное горение частиц -

$$
U_{C}=2.95 \cdot 10^{-5}\left(\frac{P}{P_{\text {атм }}}\right)^{0.8} \mathrm{MM} / \mathrm{c} .
$$

\section{Выводы}

Проведенные комплексные расчетно-экспериментальные исследования позволили получить законы диспергирования и горения высокоплотного топлива в условиях динамических давлений, реализуемых в модельной баллистической установке.

Получены картины изменения газодинамических параметров при использовании классической схемы заряжания и схемы заряжания с присоединенным зарядом из высокоплотного топлива.

Экспериментальным образом показано, что включение в состав метательного заряда высокоплотных топлив в виде ПЗ, позволяет увеличить скорость снаряда на дульном срезе на 4.9-12.7 \% по сравнению с классической схемой заряжания, при сохранении максимального давления на дно канала ствола.

\section{ЛИТЕРАТУРА}

1. Сафронов А.И., Крайнов А.Ю. Внутренняя баллистика ствольной системы с присоединенной камерой подгона. Вестник ТГПУ. 2004. Серия: Естественные и точные науки. Т. 6. № 43. С. 67-70.

2. Сафронов А.И. Повышение скоростей метания элементов для изучения поведения материалов при импульсных воздействиях // Вестник Сам. гос. техн. ун-та. Сер.: Физ.мат. науки. 2004. № 27. С. 169-172.

3. Ермолаев Б.С., Сулимов А.А., Романьков А.В., Храповский В.Е., Беляев А.А., Кроули А.Б. Конвективное горение блочных зарядов из семиканальных пороховых зерен, ингибированных поливинилбутиралем // Химическая физика. 2015. Т. 34. № 5. С. 1-11

4. Horst A.W. A Brief Journey Through the History of Gun Propulsion. ARL-TR-3671 November 2005.

5. Damse R.S., Singh A. Advanced concepts of the propulsion system for the futuristic gun ammunition // Defence Science Journal. 2003. V. 53. No. 4. P. 341-350.

6. Tompkins R.E., White K.J., Oberle W.F., Juhasz A.A. Traveling Charge Gun Firings Using Very High Burning Rate Propellants. ARMY BALLISTIC RESEARCH LAB ABERDEEN PROVING GROUND MD. Technical report - ADA203307. 35 p.

7. Ikuta $K$. Two stage travelling charge accelerator for high velocity // Sci. Technol. Energy. 2004. V. 65. No. 1. P. 25-27.

8. Xin Lu, Yanhuang Zhou, Yonggang Yu. Experimental and numerical investigations on traveling charge gun using liquid fuels // J. Appl. Mech. 2011. V. 78. Iss. 5. 051002. 6 p.

9. Бураков B.A., Буркин В.B., Ищенко А.Н. и др. Экспериментальный баллистический комплекс / Патент на изобретение № 2591132 от 20 июня 2016 г.

10. Буркин В.В, Дьячковский А.С., Ищенко А.Н., Корольков Л.В., Рогаев К.С., Саммель А.Ю., Степанов Е.Ю., Чупашев А.В. Информационно измерительный комплекс НИИ ПММ ТГУ // Сборник материалов Международной молодежной научной конференции «Актуальные проблемы современной механики сплошных сред и небесной механики», 17-19 ноября 2014. Томск: ТГУ, 2014. С. 127-128. 
11. Буркин В.В., Дьячковский А.С., Егоров А.Л., Ищенко А.Н., Корольков Л.В., Майстренко И.В., Рогаев К.С., Степанов Е.Ю., Чупашев А.В. Датчик дульной скорости // Патент на полезную модель №161396 заявка RU 2015127042 06.07.2015

12. Прибор измерения скорости в стволе ДДС 6000: паспорт. М.: Таис, 2010.8 с.

13. Хоменко Ю.П., Ищенко А.Н., Касимов В.З. Математическое моделирование внутрибаллистических процессов в ствольных системах. Новосибирск: Изд-во СО РАН, 1999. $256 \mathrm{c}$.

14. Ищенко А.Н., Касимов В.3. Математическая модель и программный комплекс для теоретического исследования внутрибаллистических процессов в ствольных системах: учеб. пособие. Томск: Издательскй дом Томского государственного университета, 2015. $70 \mathrm{c}$.

15. Серебряков M.E. Внутренняя баллистика ствольных систем и пороховых ракет. М.: Оборонгиз, 1962. 703 с.

Статья поступила 28.04.2020

Rogaev K.S., Ishchenko A.N., Burkin V.V., D’yachkovskiy A.S., Sidorov A.D., Stepanov E.Yu. (2021) A STUDY OF THE COMBUSTION OF HIGH-DENSITY PROPELLANTS IN A MODEL BALLISTIC INSTALLATION. Vestnik Tomskogo gosudarstvennogo universiteta. Matematika i mekhanika [Tomsk State University Journal of Mathematics and Mechanics]. pp. $127-138$

\section{DOI $10.17223 / 19988621 / 69 / 10$}

Keywords: internal ballistics, barrel systems, gas dynamics, travelling charge, combustion of propellants, high-density propellants.

In internal ballistics of barrel systems, a promising trend is related to the increasing of projectile muzzle velocity by means of high-energy propellants utilized as a traveling charge. The use of a loading scheme with a traveling charge allows one to increase the loading density and to redistribute the energy of powder gases in the space behind the projectile, which leads to a significant increase in the muzzle velocity of the projectile. To attain the listed advantages, it is necessary to know the laws of dispersion and combustion of the propellants used as traveling charges, providing non-digressive gas entry into the charge space.

In this work, a comprehensive experimental and theoretical study of the laws of dispersion and combustion of high-density propellants under dynamic pressures, provided in a model ballistic installation, is carried out. The main ballistic characteristics of shots are obtained, which use a classic scheme of loading with a propellant charge made of pyroxylin powder and a scheme with a traveling charge, where, in addition to the propellant charge, a high-density propellant is included. All the experiments are simulated in a software package, taking into account the presence of the high-density propellants in the propellant charge, dispersing into individual particles that burn out while moving along the barrel. As a result of comparing the calculated and experimental data, plausible patterns of the distribution of gas-dynamic parameters are obtained using the classic loading scheme and the loading scheme with a travelling high-density propellant charge.

Financial support. The study was carried out at the expenses of the grant from the Russian Science Foundation (project No. 19-79-00028).

Konstantin S. ROGAEV (Candidate of Physics and Mathematics, Research Institute of Applied Mathematics and Mechanics of Tomsk State University, Tomsk, Russian Federation). E-mail: rogaev@ftf.tsu.ru

Aleksandr N. ISHCHENKO (Doctor of Physics and Mathematics, Research Institute of Applied Mathematics and Mechanics of Tomsk State University, Tomsk, Russian Federation). E-mail: ichan@niipmm.tsu.ru 
Viktor V. BURKIN (Candidate of Physics and Mathematics, Research Institute of Applied Mathematics and Mechanics of Tomsk State University, Tomsk, Russian Federation). E-mail: v.v.burkin@mail.ru

Aleksey S. D'YACHKOVSKIY (Candidate of Physics and Mathematics, Research Institute of Applied Mathematics and Mechanics of Tomsk State University, Tomsk, Russian Federation). E-mail: lex_okha@mail.ru

Aleksey D. SIDOROV (Research Institute of Applied Mathematics and Mechanics of Tomsk State University, Tomsk, Russian Federation).E-mail: aleksid92@gmail.com

Evgeniy Yu. STEPANOV (Research Institute of Applied Mathematics and Mechanics of Tomsk State University, Tomsk, Russian Federation). E-mail: stepanov_eu@mail.ru

\section{REFERENCES}

1. Safronov A.I., Kraynov A.Yu. (2004) Vnutrennyaya ballistika stvol'noy sistemy s prisoedinennoy kameroy podgona [Interior ballistics of the barrel system with an attached matching chamber]. Vestnik Tomskogo gosudarstvennogo pedagogicheskogo universiteta Tomsk State Pedagogical University Bulletin. 43(6). pp. 67-70.

2. Safronov A.I. (2004) Povyshenie skorostey metaniya elementov dlya izucheniya povedeniya materialov pri impul'snykh vozdeystviyakh [Increasing the speed of the projection elements to study the behavior of materials under impulse actions]. Vestnik Samarskogo gosudarstvennogo tekhnicheskogo universiteta, Seriya fiziko-matematicheskie nauki. 27. pp. 169-172. DOI: 10.14498/vsgtu297.

3. Ermolaev B.S., Sulimov A.A., Roman'kov A.V., Khrapovskiy V.E., Belyaev A.A., Krouli A.B. (2015) Konvektivnoe gorenie blochnykh zaryadov iz semikanal'nykh porokhovykh zeren, ingibirovannykh polivinilbutiralem [Convective combustion of block charges from sevenchannel powder grains inhibited by polyvinyl butyral]. Khimicheskaya fizika - Russian Journal of Physical Chemistry B: Focus on Physics. 34(5). pp. 1-11. DOI: 10.7868/ S0207401X15050040.

4. Horst A.W. (2005) A Brief Journey Through the History of Gun Propulsion. DOI: 10.21236/ada441021.

5. Damse R.S., Singh A. (2003) Advanced concepts of the propulsion system for the futuristic gun ammunition. Defence Science Journal. 53(4). pp. 341-350. DOI: 10.14429/dsj.53.2279.

6. Tompkins R.E., White K.J., Oberle W.F., Juhasz A.A. (1988) Traveling charge gun firings using very high burning rate propellants. Army Ballistic Research Lab Aberdeen Proving Ground Md. Technical report - ADA203307.

7. Ikuta K. (2004) Two stage travelling charge accelerator for high velocity. Science and Technology of Energetic Materials. 65(1). pp. 25-27. DOI: 10.1002/prep.201400154.

8. Xin Lu, Yanhuang Zhou, Yonggang Yu. (2011) Experimental and numerical investigations on traveling charge gun using liquid fuels. Journal of Applied Mechanics. 78(5). pp. 051002-1051002-6. DOI: 10.1115/1.4004292.

9. Burakov V.A., Burkin V.V., Ishchenko A.N., Korol'kov L.V., Stepanov E.Yu., Agafonov S.V., Rogaev K.S., Chupashev A.V. (2016) Eksperimental'nyy ballisticheskiy kompleks [Experimental ballistic complex]. RF Patent 2591132.

10. Burkin V.V., D'yachkovskiy A.S., Ishchenko A.N., Korol'kov L.V., Rogaev K.S., Sammel' A.Yu., Stepanov E.Yu., Chupashev A.V. (2014) Informatsionno izmeritel'nyy kompleks NII PMM TGU [Data measuring complex of RIAMM]. Proceedings of International Youth Scientific Conference "Actual Problems of Modern Continuum Mechanics and Celestial Mechanics". Tomsk: RIAMM. pp. 127-128.

11. Burkin V.V., D'yachkovskiy A.S., Egorov A.L., Ishchenko A.N., Korol'kov L.V., Maystrenko I.V., Rogaev K.S., Stepanov E.Yu., Chupashev A.V. (2015) Datchik dul'noy skorosti [Muzzle velocity sensor]. RF Patent 161396.

12. (2010) Pribor izmereniya skorosti v stvole DDS 6000: passport [A device for measuring the speed in the barrel DDS 6000: passport]. Moscow: Tais. 
13. Khomenko Yu.P., Ishchenko A.N., Kasimov V.Z. (1999) Matematicheskoe modelirovanie vnutriballisticheskikh protsessov $v$ stvol'nykh sistemakh [Mathematic simulation of intraballistic processes in barrel systems]. Novosibirsk: SB RAS Publishing House.

14. Ishchenko A.N., Kasimov V.Z. (2015) Matematicheskaya model' i programmnyy kompleks dlya teoreticheskogo issledovaniya vnutriballisticheskikh protsessov v stvol'nykh sistemakh [A mathematical model and a software package for a theoretical study of intraballistic processes in barrel systems]. Tomsk: Tomsk State University Publishing House.

15. Serebryakov M.E. (1962) Vnutrennyaya ballistika stvol'nykh sistem i porokhovykh raket [Internal ballistics of barrel systems and powder rockets]. Moscow: Oborongiz.

Received: April 28, 2020 